\section{Desigualdades socioespaciais na mortalidade do adulto no Município de São Paulo*}

\section{Social inequalities in adult mortality in the City of S. Paulo}

Marcos Drumond Jr.

Programa de Aprimoramento das Informações de Mortalidade - PRO-AIM Prefeitura do Município de São Paulo.

Endereço para correspondência:

Rua Martiniano de Carvalho 676/11

Bela Vista

01321-000 São Paulo, SP - Brasil

e-mail: mdrumond@uninet.com.br

\section{Marilisa Berti de Azevedo Barros}

Departamento de Medicina Preventiva e Social

Faculdade de Ciências Médicas

Universidade Estadual de Campinas - UNICAMP

Cidade Universitária - Campinas - São Paulo

e-mail: marilisa@obelix.unicamp.br

\section{Resumo}

O aumento da importância da mortalidade do adulto tem sido acompanhado de uma grande produção científica sobre o tema nos países centrais. Nestes estudos, as desigualdades sociais têm sido avaliadas. Nos países periféricos, apesar de também se observar o crescimento da sua importância, têm sido realizados poucos estudos, em especial no que se refere às desigualdades sociais na sua geração e distribuição. Este trabalho tem como objetivo a análise das desigualdades sociais na mortalidade do adulto no município de São Paulo, buscando-se a apreensão dessa desigualdade pelo estudo dos diferenciais no espaço urbano. Este é analisado pela definição e delimitação de áreas socioambientais homogêneas. Nos resultados observa-se distribuição desigual na mortalidade do adulto no município de São Paulo, constatada nos diferentes indicadores analisados e para a maioria das causas de morte analisadas, geralmente em prejuízo das populações vivendo em áreas de piores condições socioambientais. O padrão da mortalidade do adulto, no Município de São Paulo, mostra forte participação de doenças crônicas não transmissíveis e das decorrentes de violências. São discutidas algumas dimensões de determinação dos perfis encontrados ressaltando as distribuições diferenciadas dos fatores de risco, do acesso à assistência à saúde, da exposição a riscos no ambiente urbano e das condições econômicas e sociais da população.

Palavras-chave: Mortalidade. Adulto. Fatores socioeconômicos. Iniqüidade social. Distribuição espacial.

* Extraído da dissertação de mestrado "Desigualdades socioespaciais na mortalidade evitável do adulto no município de São Paulo", apresentada ao Curso de Pós-graduação em Saúde Coletiva da Faculdade de Ciências Médicas da UNICAMP em 6/12/96. Trabalho realizado com apoio financeiro através de bolsa de estudo do Conselho Nacional do Desenvolvimento Científico e Tecnológico (CNPq). Processo No $132407 / 94-9$ 


\section{Abstract}

Mortality among adults has been increasingly analyzed in developed countries because it has become relatively more important than mortality in other age groups. Social inequalities have been assessed in these studies. In developing countries these issues have not been sufficiently analyzed. This article aims to detect social differences in mortality among adults in City of São Paulo, between 1990 and 1992. The urban area of the City of São Paulo was divided into four socioeconomic homogeneous areas. This division was based on socioeconomic variables obtained from the 1991 demographic census. Comparing the four areas, the results showed important differences in several death indexes and death rates. Death rates for most basic causes were higher in underprivileged areas. The main causes of adult mortality were cardiovascular diseases, tumors, accidents and violence. Some possible groups of determinants that could explain the differences in the mortality pattern found in the City of São Paulo were discussed: different prevalence of known risk factors for chronic diseases; different access to adequate medical care; different quality of urban environment, and different socioeconomic levels of the population living in different areas of the city.

Keywords: Mortality. Adult. Socioeconomic factors. Social iniquity. Residence characteristics.

\section{Introdução}

Tem aumentado a importância dos problemas de saúde do adulto nos perfis de morbi-mortalidade das populações. Esse aumento decorre da redução da mortalidade infantil, do envelhecimento populacional e do aumento da incidência de alguns agravos, como aids e violência. Com o predomínio dos agravos que acometem adultos e idosos, cresce a produção científica sobre o tema nos países centrais ${ }^{1,2}$, mas ela continua insuficiente nos países em desenvolvimento. Murray e Feachem ${ }^{3}$ consideram que prevalece a crença equivocada de que seriam pequenas as diferenças nos riscos de morte de adultos entre os países industrializados e aqueles em desenvolvimento. E contestam essa impressão, estimando a ocorrência anual de 8 milhões de mortes evitáveis de adultos na América Latina, Ásia e África, tendo os níveis do Japão como referência.

A diferenciação dos níveis e padrões de mortalidade entre grupos sociais de uma mesma sociedade tem sido detectada em diversos estudos, com o uso de diferentes abordagens analíticas e diversas variáveis sociais. Nos países periféricos, a maioria dos estudos sobre desigualdade em saúde enfocava os temas da mortalidade infantil e das mortes por doenças infecciosas ${ }^{4,5}$, sendo mais recentes os estudos que abordam as desigualdades sociais tendo por objeto a mortalidade do adulto.

Nos países centrais há uma significativa produção sobre o tema das desigualdades sociais em saúde. Um marco nessa produção é o Black Report ${ }^{6}$, que estimulou análises realizadas em diversos países. Esse estudo mostrou que os riscos de morte de trabalhadores manuais são maiores que os dos trabalhadores não-manuais, apesar da universalidade da atenção à saúde na GrãBretanha. Outros estudos também mostram grandes diferenças relativas aos níveis, padrões e tendências da mortalidade do adulto, revelando que, mesmo em países com alto padrão de vida e em que o acesso aos serviços de saúde é universal e aparen- 
temente não-desigual, como a Suécia e a Suíça, as diferenças estão presentes, em especial entre adultos ${ }^{7-10}$. Esses estudos revelam que há maiores riscos de morte nos segmentos da população constituídos por trabalhadores manuais, por imigrantes, por grupos sociais com baixa escolaridade e renda, por pessoas sem casa própria, por famílias chefiadas por mulheres não-casadas, por desempregados e por aposentados.

Análises contidas na atualização do Black Report ${ }^{11}$ confirmam a tendência de distanciamento nos riscos de morte entre classes sociais, verificando que a atenção destinada à saúde não foi capaz de reduzir as desigualdades reveladas na década anterior. São observados grandes diferenciais nos riscos de morte por diversas doenças crônicas não-transmissíveis, com altas taxas nos trabalhadores manuais e não-qualificados, o que reforça o questionamento de conceitos anteriormente usados, como o de "doenças da afluência ou de executivos". Nas classes sociais mais bem posicionadas $^{12}$, apresentaram maiores taxas padronizadas de mortalidade apenas, entre os homens, o melanoma maligno e, entre as mulheres, o melanoma maligno, a leucemia linfóide crônica e os cânceres de mama e cérebro.

Nos Estados Unidos, as desigualdades sociais na mortalidade do adulto são estudadas, em geral, com a utilização das variáveis raça e etnia. Keil e col. ${ }^{13}$ mostram que a razão entre os coeficientes de mortalidade de negros e brancos foi de 1,6 para o conjunto das 15 principais causas de morte no país em 1989. Os coeficientes dos negros são maiores em 13 destas causas. Os maiores diferenciais foram observados para homicídios (razão de 6,6) e aids (3,3). Os autores mostram a ausência de diferença significativa nos riscos de morte entre brancos e negros após ajustar para os efeitos do nível educacional e ocupacional, concluindo que é a classe social que basicamente responde pelas diferenças de mortalidade entre os grupos étnicos.

No Brasil, é mais recente a abordagem das desigualdades sociais na mortalidade do adulto. Entre os estudos realizados, Stephens e col. ${ }^{14}$ discutem diferenciais intra-urbanos da mortalidade no município de São Paulo, mostrando que, nas idades de 15 a 44 anos, o coeficiente de mortalidade por causas externas, nas áreas de moradia da população em piores condições socioambientais, foi mais que o dobro do das áreas de melhor condição. Nas idades de 45 a 64 anos esse estudo indica excesso de mortes por doenças cerebrovasculares, hipertensivas e acidentes de trânsito nos moradores da área de pior condição socioambiental . Lessa ${ }^{15}$, estudando a mortalidade por doenças cerebrovasculares segundo região do país, mostra que as mortes por essas causas nas regiões mais pobres ocorrem mais precocemente. A morte de adultos, especialmente dos jovens, é em geral abordada nos estudos dedicados às mortes por causas externas ${ }^{16}$. Mais recentemente os homicídios têm sido avaliados segundo grupos sociais ${ }^{17,18}$.

O espaço sociogeográfico tem sido tradicionalmente utilizado em epidemiologia, em especial no estudo das doenças infecciosas. Alguns pesquisadores têm discutido um entendimento totalizador do conceito de espaço, definido como espaço histórico, construído pelo homem. As necessidades econômicas seriam determinantes importantes da forma de organização do espaço. Barreto ${ }^{19}$ considera que, apesar de os fenômenos do processo saúde-doença ocorrerem no interior de um espaço que apresenta, antes de tudo, características físicas, climáticas e ecológicas, esse espaço passa a ter importância humana e social, portanto histórica, no momento em que o homem se faz presente, sintetizando que o tempo histórico incorpora a paisagem no espaço.

Breilh e col. ${ }^{20}$ discutem que "a contradição entre os caracteres benéficos ou destrutivos que podem ter os elementos geoecológicos expressa também as contradições da ordem social estabelecida". Exemplificam que nem a água, nem a umidade, nem o calor, nem os microorganismos e mosquitos são "em si" epidemiologicamente destrutivos, mas na sua 
interação com formas de exploração do ambiente podem desencadear processos deteriorantes para a saúde. Sob a lógica predominante do crescimento do lucro, o aumento da produção industrial pode levar à poluição do ar e da água, que vão se incorporar no consumo das classes urbanas como processos destrutivos. Da mesma forma, as mudanças climáticas como o calor ou o frio excessivos provocam impactos em populações carentes, em função de inadequações no vestuário ou moradia.

O espaço social de uma cidade, ao expressar as condições de vida dos segmentos que o ocupam, representa uma instância da sociedade que contém as várias dimensões da estrutura social - econômicas, políticas e ideológicas - que nele se reproduzem $^{21}$. O espaço encontra-se articulado a/contido nessas outras instâncias. Paim ${ }^{21}$ considera que as condições de vida específicas de cada classe social podem ser consideradas mediadoras de determinantes estruturais. Acrescenta que a operacionalização do conceito de condições de vida através de variáveis e indicadores selecionados, considerando os grupos humanos dispostos em diferentes espaços da cidade, permite uma aproximação da realidade que preserva em parte sua complexidade.

Considerar a forma de organização social do espaço constitui uma das estratégias para o entendimento da ocorrência e distribuição de agravos à saúde. Além disso, como os serviços de saúde são organizados em base espacial, esses conhecimentos podem contribuir para a adequação das ações de saúde às necessidades diferenciadas da população.

Illsley ${ }^{22}$, revisando a metodologia dos estudos sobre desigualdades e saúde na Europa, afirma que as análises da mortalidade, referenciadas no espaço e relacionadas a dados sociais, dão contribuições importantes para o conhecimento das determinações dos perfis e taxas encontrados. Afirma, ainda, que as análises utilizando os diferenciais espaciais podem ser um complemento importante daquelas utilizando grupos ocupacionais, com a vantagem de se aplicarem a ambos os sexos e a todos os grupos de idade.

Por outro lado, algumas limitações dificultam o uso do espaço social como categoria de análise. As principais desvantagens dos estudos baseados em áreas locais e regionais são: os limites administrativos não coincidem necessariamente com áreas significativas do ponto de vista social, e a possibilidade de existência de grande e variável heterogeneidade interna ${ }^{11,22}$. Outro aspecto a considerar é a dinâmica de mobilidade da população entre áreas, produzindo mudanças em sua composição social, às vezes em curto intervalo de tempo ${ }^{22}$.

Considerando-se a importância crescente da mortalidade do adulto, a necessidade do conhecimento dos diferenciais sociais nessa mortalidade e os potenciais do uso do espaço sociogeográfico para a apreensão das desigualdades sociais em saúde, tem-se como objetivo deste estudo a análise das desigualdades sociais na mortalidade do adulto no município de São Paulo, buscando-se a apreensão dessa desigualdade pelo estudo dos diferenciais no espaço urbano.

\section{Material e Métodos}

\section{Fontes de dados e indicadores utilizados}

Foram utilizados dados dos óbitos de residentes no município de São Paulo dos anos de 1990, 1991 e 1992, obtidos da Fundação SEADE. Foram analisadas as variáveis sexo, idade, distrito de paz de residência e causa básica da morte. Para o cálculo dos coeficientes foram utilizados dados populacionais do Censo Demográfico de 1991 do Instituto Brasileiro de Geografia e Estatística (IBGE), com estimativas populacionais para $1^{\circ}$ de julho de 1991, elaboradas pela Fundação SEADE. Para cálculo dos indicadores específicos por idade foram utilizadas as seguintes faixas etárias: 15-24, 25-34, 35-44, 45-54 e 55-64 anos.

Os indicadores calculados foram:

1. Mortalidade proporcional dos adultos (15-64 anos) em relação ao total de óbitos. 
2. Mortalidade proporcional precoce dos adultos (15-34 anos) em relação à mortalidade dos adultos (15-64 anos).

3. Coeficiente padronizado dos anos potenciais de vida perdidos entre 15 e 65 anos.

4. Probabilidade de morte, calculada pelas tábuas de vida da população do município de São Paulo. Foi utilizado o 50q15, que informa da probabilidade de morrer até os 65 anos, dado que estava vivo aos 15 anos.

5. Coeficientes de mortalidade específicos por sexo e faixa etária.

6. Coeficiente de 15-64 anos padronizado pelo método direto, utilizando como padrão a população municipal do ano censitário de 1991.

7. Coeficientes de mortalidade por causa básica, padronizados por idade pelo método direto, usando a população do município como padrão.

Para realizar a comparação dos índices, coeficientes e probabilidades entre as áreas homogêneas (descritas a seguir) foram utilizados:

1. Razões entre índices, probabilidades e coeficientes específicos por idade, observados na área homogênea 4 em relação aos observados na área 1 , considerada como referência.

2. Razão entre os coeficientes de mortalidade por causas específicas e por todas as causas, padronizados por idade pelo método direto, das regiões de piores níveis socioambientais (áreas 2, 3 e 4) e aqueles da região de melhor nível (área 1).

Todos os indicadores calculados referem-se às médias observadas para o triênio 1990 - 1992. Foi testada a significância da diferença entre os coeficientes de mortalidade, padronizados pelo método direto, segundo causas básicas de morte entre as áreas 4 e 1 . $\mathrm{O}$ teste foi realizado de acordo com método proposto por Armitage ${ }^{23}$.

\section{Causas básicas da morte}

As causas de morte foram codificadas e classificadas segundo a CID-9 (9ª revisão da Classificação Internacional de Doenças 1975). Foi utilizada a lista básica de tabulação proposta pela CID-9 segundo categorias e subcategorias.

Foram incluídas para análise neste estudo todas as causas básicas que se encontravam entre as cinco causas de morte de maior freqüência, em cada faixa etária decenal entre 15 e 64 anos, para cada sexo. Esse procedimento levou à inclusão das seguintes causas:

- no sexo masculino: homicídios, doenças isquêmicas do coração, aids, acidentes de trânsito com veículo a motor, doenças cerebrovasculares, doenças da circulação pulmonar e outras formas de doenças do coração, cirrose hepática e doença crônica do fígado, pneumonias, câncer de pulmão, suicídios e afogamentos.

- no sexo feminino: doenças cerebrovasculares, doenças isquêmicas do coração, doenças da circulação pulmonar e outras formas de doenças do coração, câncer de mama, pneumonias, acidentes de trânsito com veículo a motor, diabetes mellitus, aids, homicídios e suicídios.

A essas causas foram acrescentadas outras, consideradas evitáveis através de intervenções preventivas e/ou assistenciais, incluídas na relação proposta por Rutstein DD e col. ${ }^{24}$ e Westerling e col. ${ }^{10}$, e que apresentavam freqüência de ao menos 150 óbitos no município no ano de 1991. Esses procedimentos levaram à adição das seguintes causas: tuberculose, doença de Chagas, cânceres de esôfago, estômago, cólon, fígado, laringe, colo uterino, útero porção não especificada, síndrome de dependência do álcool, doença reumática crônica do coração, doenças hipertensivas, miocardiopatias, insuficiência cardíaca, doenças pulmonares obstrutivas crônicas e insuficiência renal.

\section{Áreas homogêneas}

Foi utilizada a divisão espacial baseada 
em indicadores socioeconômicos da pesquisa "Saúde e ambiente, diferenciais intraurbanos da mortalidade no município de São Paulo" ${ }^{14}$. Os autores utilizaram, para a construção das áreas homogêneas, os seguintes indicadores: proporção de adultos analfabetos ou com primário incompleto como nível de escolaridade em 1990, renda média (em salários mínimos) per capita em 1990, densidade habitacional média (pessoas por domicílio) em 1991, proporção de domicílios ligados à rede de esgotos e consumo médio de água per capita em 1993. Foi construído um índice composto pela síntese das variáveis que, ordenado, produziu quatro zonas socioambientais homogêneas, denominadas neste estudo de:

- área homogênea 1, composta dos distritos com as melhores condições socioambientais, nos quais viviam $9,2 \%$ da população municipal;

- área homogênea 2, intermediária superior, com $13,5 \%$ da população;

- área homogênea 3, intermediária inferior, com $33,5 \%$ da população;

- área homogênea 4, composta dos distritos com as piores condições socioambientais, nos quais viviam $43,5 \%$ da população.

Essas áreas apresentam uma distribuição relativamente concêntrica. A organização socioespacial do município de São Paulo representa parte da história da sua estruturação como centro da metrópole mais dinâmica, do ponto de vista econômico, do capitalismo periférico brasileiro. A região central da cidade é dividida em duas áreas. Uma se expande para sul e oeste onde fica a área 1 e se consolida como aquela onde moram as populações de classe média e alta. É uma área com grande dinamismo na economia imobiliária, de consumo e de gerenciamento do capital. A outra área se expande para norte e leste, onde se encontra o centro velho da cidade. Este, que compõe uma parte central da área 2, constitui um centro econômico marcado pela decadência e contém espaços em processo de "encortiçamento" progressivo, além de abrigar um amplo mercado ambulante e ilegal de trabalhadores informais e de excluídos sociais. As áreas 3 e 4 expressam a expansão periférica das populações de média e baixa renda. Em todas essas áreas a heterogeneidade é presente e marca dificuldades metodológicas para o estudo que exigiriam áreas menores para aumentar a homogeneidade. No entanto, em qualquer nível de agregação, restam heterogeneidades internas, decorrentes da complexidade da estruturação e da dinâmica social da cidade. As médias das áreas irão representar diferentes níveis de condições socioeconômicas e socioambientais, com a perspectiva de satisfazer aos propósitos deste estudo: contribuir para o conhecimento das desigualdades sociais na mortalidade do adulto.

\section{Resultados}

\section{Desigualdades socioespaciais segundo alguns indicadores de mortalidade do adulto}

Observa-se na Tabela 1, referente ao sexo masculino, que os indicadores da área homogênea 4 são de $24 \%$ a $58 \%$ superiores aos observados na área 1 . Na Tabela 2 , referente ao sexo feminino, verifica-se que os indicadores da área 4 são maiores que os da área 1 em $37 \%$ a $78 \%$. Na comparação entre as Tabelas 1 e 2, destacam-se as diferenças expressivas existentes nos indicadores entre os sexos.

As análises dos coeficientes de mortalidade específicos por idade e do coeficiente de 15 a 64 anos padronizado por idade (Tabelas 3 e 4) confirmam essas diferenças. Os coeficientes de mortalidade dos homens da área de melhor nível socioeconômico ainda são maiores do que os das mulheres da área de pior nível. O coeficiente de mortalidade do adulto do sexo masculino na área 4 foi $30 \%$ maior que na área 1 (Tabela 3) e, do feminino, 50\% maior (Tabela 4). No sexo masculino, as razões entre os coeficientes de mortalidade específicos por idade das áreas 4 e 1 variaram de 1,1 a 1,3 nas faixas 
Tabela 1 - Indicadores de mortalidade do adulto segundo áreas homogêneas no sexo masculino. Município de São Paulo, 1990-92.

Table 1 - Mortality indicators among male adults by homogeneous areas. São Paulo City, 1990-92.

\begin{tabular}{|c|c|c|c|c|c|c|}
\hline \multirow[b]{2}{*}{ Indicador } & \multirow{2}{*}{$\begin{array}{l}\text { nunicípio de } \\
\text { São Paulo }\end{array}$} & \multicolumn{4}{|c|}{ áreas homogêneas } & \multirow{2}{*}{$\begin{array}{c}\text { razão } \\
\text { área 4/ área } 1\end{array}$} \\
\hline & & área 1 & área 2 & área 3 & área 4 & \\
\hline $\begin{array}{l}\text { mortalidade proporcional } \\
\text { de } 15 \text { a } 64 \text { anos }\end{array}$ & 57,1 & 43,4 & 49,6 & 55,3 & 62,8 & 1,45 \\
\hline $\begin{array}{l}\text { proporção de mortes de } \\
15-34 \text { em relação } \\
\text { à } 15-64 \text { anos }\end{array}$ & 34,9 & 25,3 & 28,4 & 31,5 & 40,0 & 1,58 \\
\hline $\begin{array}{l}\text { anos potenciais de vida } \\
\text { perdidos coeficiente } \\
\text { por } 10.000\end{array}$ & 160,3 & 128,3 & 139,9 & 145,4 & 176,7 & 1,38 \\
\hline $\begin{array}{l}\text { probabilidade de morte } \\
\text { entre } 15 \text { e } 64 \text { anos }\end{array}$ & 0,38 & 0,33 & 0,36 & 0,36 & 0,41 & 1,24 \\
\hline
\end{tabular}

Fonte dos dados brutos: Fundação SEADE e IBGE

Raw data source: Fundação SEADE and IBGE

Tabela 2 - Indicadores de mortalidade do adulto segundo áreas homogêneas no sexo feminino. Município de São Paulo, 1990-92.

Table 2 - Mortality indicators among female adults by homogeneous areas. São Paulo City, 1990-92.

\begin{tabular}{|c|c|c|c|c|c|c|}
\hline \multirow[b]{2}{*}{ Indicador } & \multirow{2}{*}{$\begin{array}{l}\text { município de } \\
\text { São Paulo }\end{array}$} & \multicolumn{4}{|c|}{ áreas homogêneas } & \multirow{2}{*}{$\begin{array}{c}\text { razão } \\
\text { área 4/ área } 1\end{array}$} \\
\hline & & área 1 & área 2 & área 3 & área 4 & \\
\hline $\begin{array}{l}\text { mortalidade proporcional } \\
\text { de } 15 \text { a } 64 \text { anos }\end{array}$ & 36,2 & 24,6 & 28,6 & 34,9 & 43,8 & 1,78 \\
\hline $\begin{array}{l}\text { proporção de mortes de } \\
15-34 \text { em relação } \\
\text { à } 15-64 \text { anos }\end{array}$ & 19,4 & 14,4 & 17,4 & 17,2 & 21,6 & 1,50 \\
\hline $\begin{array}{l}\text { anos potenciais de vida } \\
\text { perdidos coeficiente } \\
\text { por } 10.000\end{array}$ & 48,5 & 38,0 & 42,6 & 44,8 & 52,2 & 1,37 \\
\hline $\begin{array}{l}\text { probabilidade de morte } \\
\text { entre } 15 \text { e } 64 \text { anos }\end{array}$ & 0,19 & 0,13 & 0,16 & 0,18 & 0,22 & 1,69 \\
\hline
\end{tabular}

Fonte dos dados brutos: Fundação SEADE e IBGE

Raw data source: Fundação SEADE and IBGE

etárias de 25 a 64 anos. Na faixa de 15 a 24 anos, a razão atinge o valor de 1,9. As razões entre as áreas foram maiores no sexo feminino nas faixas decenais de 25 a 64 anos $(1,5,1,5,1,6$ e 1,6, respectivamente), ficando em 1,2 no grupo de 15 a 24 anos. Esses valores mostram uma maior desigualdade no sexo feminino que no masculino, com exceção do grupo de 15 a 24 anos.

\section{Desigualdades socioespaciais segundo causas de morte}

A Tabela 5 mostra as dez principais causas de morte em cada uma das áreas homo- gêneas segundo o sexo. Observa-se, entre os homens, que, enquanto na área 1 a principal causa de morte é a aids, nas demais são os homicídios, que chegam, na área 4 , a representar quase $1 / 4$ do total de mortes. A aids reduz sua importância no perfil de mortalidade nas áreas de piores condições socioambientais, chegando à quinta posição na área 4. Em geral, as principais causas de morte são semelhantes em todas as áreas, alterando sua posição na ordenação. Destaca-se o surgimento do alcoolismo nas áreas 3 e 4, e das doenças hipertensivas na área 4 entre as dez principais causas. No sexo feminino, o câncer de mama é a prin- 
Tabela 3 - Coeficientes de mortalidade por 10.000 habitantes, do adulto do sexo masculino de 15 a 64 anos, segundo faixa etária e áreas homogêneas. Município de São Paulo, 1990-92.

Table 3 - Death rates among male adults by age groups and homogeneous areas. São Paulo City, 1990-92.

\begin{tabular}{ccccccc}
\hline Faixa etária & município de & \multicolumn{4}{c}{ áreas homogêneas } & razão \\
\cline { 3 - 6 } (em anos) & São Paulo & área 1 & área 2 & área 3 & área 4 nnnnnn área 4/ área 1 \\
\hline $15-24$ & 39,4 & 23,6 & 31,7 & 40,7 & 45,1 & 1,9 \\
$25-34$ & 46,6 & 40,2 & 40,7 & 42,7 & 49,7 & 1,2 \\
$35-44$ & 61,6 & 59,0 & 58,3 & 57,4 & 64,9 & 1,1 \\
$45-54$ & 108,9 & 93,2 & 101,5 & 106,5 & 115,3 & 1,2 \\
$55-64$ & 214,9 & 185,2 & 201,4 & 208,0 & 232,3 & 1,3 \\
$15-64 *$ & 70,3 & 59,0 & 63,8 & 65,9 & 76,1 & 1,3 \\
\hline
\end{tabular}

Fonte dos dados brutos: Fundação SEADE e IBGE

Raw data source: Fundação SEADE and IBGE

* coeficiente de 15 a 64 anos padronizado pelo método direto, usando como padrão a população do município em 1991

* 15 to 65 age group rate using the direct method of age standardization, and the total 1991 population of the city as standard

Tabela 4 - Coeficientes de mortalidade por 10.000 habitantes, do adulto do sexo feminino de 15 a 64 anos, segundo faixa etária e áreas homogêneas. Município de São Paulo, 1990-92.

Table 4 - Death rates among female adults by age groups and homogeneous areas. São Paulo City, 1990-92.

\begin{tabular}{ccrrrrr}
\hline Faixa etária & município de & \multicolumn{4}{c}{ áreas homogêneas } & razão \\
\cline { 3 - 5 } (em anos) & São Paulo & área 1 & área 2 & área 3 & área 4 & área 4/ área 1 \\
\hline $15-24$ & 7,8 & 6,3 & 7,4 & 6,5 & 7,8 & 1,2 \\
$25-34$ & 11,6 & 8,2 & 10,4 & 10,8 & 12,5 & 1,5 \\
$35-44$ & 22,5 & 16,8 & 18,2 & 20,7 & 24,7 & 1,5 \\
$45-54$ & 49,8 & 38,8 & 41,3 & 47,0 & 60,6 & 1,6 \\
$55-64$ & 110,4 & 82,9 & 91,0 & 106,7 & 133,4 & 1,6 \\
$15-64^{*}$ & 27,8 & 21,0 & 23,3 & 26,1 & 32,2 & 1,5 \\
\hline
\end{tabular}

Fonte dos dados brutos: Fundação SEADE e IBGE

Raw data source: Fundação SEADE and IBGE

* coeficiente de 15 a 64 anos padronizado pelo método direto, usando como padrão a população do município em 1991

*15 to 65 age group rate using the direct method of age standardization, and the total 1991 population of the city as standard

cipal causa de morte na área 1, enquanto nas demais são as doenças cerebrovasculares. O câncer de mama vai perdendo importância nas outras áreas, chegando à quinta posição na área 4 . Também entre as mulheres as principais causas são, no geral, semelhantes entre as áreas. Destacase o câncer de pulmão, que aparece apenas na área 1, e o homicídio, que aparece nas áreas 2 e 4, entre as dez principais causas básicas de morte.

Observa-se nas Tabelas 6 e 7 que o risco de morrer pelas diferentes causas básicas apresenta grande variação entre as áreas homogêneas. Para a maioria das causas de morte analisadas, os coeficientes são crescentes da área 1 para a área 4, como pode ser apreendido pelos riscos relativos. Esse gradiente crescente pode ser observado em 11 causas de morte no sexo masculino e 24 no feminino. Destacam-se, com diferenças significativamente maiores na área $4 \mathrm{em}$ relação à área 1, os coeficientes de mortalidade pelas seguintes causas:

- entre os homens - doença de Chagas, doenças hipertensivas, doenças da circulação pulmonar e outras formas de doenças do coração, miocardiopatias, 
Tabela 5 - Mortalidade proporcional pelas 10 principais causas entre 15 e 64 anos, segundo área homogênea e sexo. Município de São Paulo, 1991.

Table 5 - Main causes of deaths among adults by sex and homogeneous areas. São Paulo City, 1991

\begin{tabular}{|c|c|c|c|c|}
\hline \multicolumn{2}{|r|}{ masculino } & \multicolumn{3}{|c|}{ feminino } \\
\hline & causa & $\%$ & causa & $\%$ \\
\hline & \multicolumn{4}{|l|}{ área 1} \\
\hline 1 & aids & 17,4 & câncer de mama & 9,1 \\
\hline 2 & infarto do miocárdio & 10,4 & doenças cerebrovasculares & 8,8 \\
\hline 3 & homicídios & 8,0 & infarto do miocárdio & 7,0 \\
\hline 4 & acidentes de trânsito & 5,8 & d. circ. pulmonar e outras d. coração & 5,1 \\
\hline 5 & doenças cerebrovasculares & 4,1 & acidentes de trânsito & 4,2 \\
\hline 6 & d. circ. pulmonar e outras d. coração & 3,7 & aids & 4,0 \\
\hline 7 & pneumonias & 3,7 & pneumonias & 3,6 \\
\hline 8 & doença crônica fígado e cirrose & 3,5 & câncer de pulmão & 2,3 \\
\hline 9 & câncer de pulmão & 3,0 & diabetes & 2,2 \\
\hline \multirow[t]{2}{*}{10} & outras doenças isquêmicas coração & 2,5 & doenças hipertensivas & 2,1 \\
\hline & \multicolumn{4}{|l|}{ área 2} \\
\hline 1 & homicídios & 12,2 & doenças cerebrovasculares & 8,4 \\
\hline 2 & aids & 10,3 & cancer de mama & 8,4 \\
\hline 3 & infarto do miocárdio & 9,9 & infarto do miocárdio & 8,4 \\
\hline 4 & acidentes de trânsito & 5,7 & d. circ. pulmonar e outras d. coração & 5,8 \\
\hline 5 & doenças cerebrovasculares & 5,6 & pneumonias & 3,6 \\
\hline 6 & pneumonias & 4,5 & aids & 3,5 \\
\hline 7 & doença crônica do fígado e cirrose & 4,5 & acidentes de trânsito & 3,4 \\
\hline 8 & d. circ. pulmonar e outras d. coração & 4,0 & diabetes & 3,1 \\
\hline 9 & câncer de pulmão & 2,1 & doenças hipertensivas & 2,7 \\
\hline \multirow[t]{2}{*}{10} & outras doenças isquêmicas coração & 1,9 & homicídios & 2,1 \\
\hline & \multicolumn{4}{|l|}{ área 3} \\
\hline 1 & homicídios & 13,7 & doenças cerebrovasculares & 10,8 \\
\hline 2 & infarto do miocárdio & 9,2 & infarto do miocárdio & 8,2 \\
\hline 3 & aids & 8,3 & d. circ. pulmonar e out. d. coração & 6,7 \\
\hline 4 & doenças cerebrovasculares & 6,5 & câncer de mama & 5,4 \\
\hline 5 & acidentes de trânsito & 6,3 & pneumonias & 4,1 \\
\hline 6 & pneumonias & 4,6 & diabetes & 4,0 \\
\hline 7 & d. circ. pulmonar e outras d. coração & 4,3 & acidentes de trânsito & 3,6 \\
\hline 8 & doença crônica do fígado e cirrose & 4,2 & aids & 3,2 \\
\hline 9 & câncer de pulmão & 2,0 & doenças hipertensivas & 2,7 \\
\hline \multirow[t]{2}{*}{10} & alcoolismo & 1,9 & insuficiência cardíaca & 2,4 \\
\hline & \multicolumn{4}{|l|}{ área 4} \\
\hline 1 & homicídios & 23,8 & doenças cerebrovasculares & 12,3 \\
\hline 2 & infarto do miocárdio & 7,3 & infarto do miocárdio & 7,9 \\
\hline 3 & acidentes de trânsito & 6,4 & d. circ. pulmonar e outras d. coração & 6,7 \\
\hline 4 & doenças cerebrovasculares & 6,3 & pneumonias & 4,3 \\
\hline 5 & aids & 5,2 & câncer de mama & 4,2 \\
\hline 6 & pneumonias & 3,9 & acidentes de trânsito & 3,6 \\
\hline 7 & d. circ. pulmonar e outras d. coração & 3,8 & diabetes & 3,5 \\
\hline 8 & doença crônica do fígado e cirrose & 3,7 & doenças hipertensivas & 3,5 \\
\hline 9 & alcoolismo & 2,0 & homicídios & 3,3 \\
\hline 10 & doenças hipertensivas & 1,9 & aids & 2,7 \\
\hline
\end{tabular}

Fonte dos dados brutos: Fundação SEADE e IBGE

Raw data source: Fundação SEADE and IBGE 
insuficiência cardíaca, doenças cerebrovasculares, pneumonias, alcoolismo, cirrose hepática, afogamentos e homicídios;

- entre as mulheres - tuberculose, doença de Chagas, diabetes, doenças hipertensivas, infarto agudo do miocárdio, doenças da circulação pulmonar e outras formas de doenças do coração, insuficiência cardíaca, doenças cerebrovasculares e pneumonias.

Essas foram algumas das causas de morte de adultos que apresentaram os maiores diferenciais socioespaciais no município. Observa-se que, no sexo feminino, se destacam as doenças cardiovasculares, diabetes e algumas infecciosas, enquanto no masculino aparecem causas externas e doenças relacionadas ao consumo de álcool, além das doenças do aparelho circulatório e algumas infecciosas.

As causas de morte que apresentaram um gradiente inverso, isto é, os riscos de morte foram maiores nas regiões de melhores condições socioambientais, são: câncer de cólon, câncer de pulmão, aids e suicídio, em ambos os sexos, o câncer de mama, no feminino, e o câncer de fígado e outras doenças isquêmicas do coração, no masculino. Dessas causas, apenas a aids no sexo masculino apresentou diferença estatisticamente significante entre as áreas 4 e 1 .

\section{Discussão e Conclusões}

O presente estudo registra distribuição desigual na mortalidade do adulto no município de São Paulo, constatada nos diferentes indicadores analisados. Estes revelam valores mais elevados nas áreas de piores condições socioambientais, seja quando indicam a probabilidade de morte, os anos potenciais de vida perdidos, a concentração de mortes de adultos em relação ao total de mortes, a concentração de mortes mais precoces ou quando estimam as taxas de mortalidade em ambos os sexos.

A análise da mortalidade segundo causas mostrou diferenças sociais importantes nos coeficientes de mortes por doenças infecciosas (tais como aids, pneumonias, tuberculose), por doenças crônicas nãotransmissíveis (doenças cardiovasculares e alguns cânceres, entre outras) e por causas externas. Com poucas exceções, os coeficientes são maiores nas regiões de piores condições socioambientais.

Algumas considerações podem ser feitas com respeito aos coeficientes de algumas causas de morte que se apresentaram mais elevados nas regiões de melhores condições socioambientais. Os resultados deste estudo apontam maior mortalidade por aids na área 1 e coeficiente por esta causa, no sexo masculino, mais de 10 vezes superior ao feminino. As formas predominantes de transmissão da doença no início da epidemia, bem como a concentração de "trabalhadores do sexo” residentes em áreas centrais da cidade ${ }^{25}$, poderiam responder em parte pela distribuição espacial observada no período deste estudo. A dinâmica da epidemia, com a tendência de periferização e homogeneização entre os sexos, alteraria nos anos seguintes essa distribuição da mortalidade ${ }^{26}$. Vale destacar que o centro velho da cidade, marcado pela decadência econômica, presença de cortiços, de comércio atacadista e ambulante e de prostituição, faz parte das áreas homogêneas 1 e 2, que apresentam, na média, as melhores condições socioambientais.

Com relação ao suicídio, ressalta-se que o padrão observado no Brasil é de coeficientes maiores em regiões e cidades do Centro-Sul, mostrando que o melhor nível socioeconômico médio se relaciona aos maiores coeficientes ${ }^{27}$. Os resultados encontrados para o câncer de mama confirmam alguns estudos que já apresentavam esse padrão de diferenciais ${ }^{12}$. A distribuição espacial do câncer de pulmão é de análise mais complexa, uma vez que se associa à distribuição e dinâmica da prevalência do hábito de fumar nos diferentes segmentos sociodemográficos da população, nas décadas que precederam o estudo. Por outro lado, a poluição atmosférica é maior nas áreas centrais da cidade de São Paulo, es- 
Tabela 6 - Coeficientes de mortalidade* da área 1 e riscos relativos entre coeficientes* das áreas homogêneas 2, 3 e 4 em relação aos da área 1, segundo causas básicas, no sexo masculino. Município de São Paulo, 1990-92.

Table 6 - Ratios of the mortality rates for homogeneous areas 2, 3 and 4 to the rate for area 1, by causes of death (males 15-64). São Paulo City, 1990-92.

\begin{tabular}{|c|c|c|c|c|}
\hline \multirow[b]{2}{*}{ causa de morte } & área 1 & área 2 & área 3 & área 4 \\
\hline & coeficiente & $\mathrm{rr}$ & $\mathrm{rr}$ & $\mathrm{rr}$ \\
\hline todas as causas & 590,0 & 1,08 & 1,12 & 1,29 \\
\hline tuberculose & 8,7 & 1,20 & 1,23 & 1,54 \\
\hline doença de Chagas** & 2,9 & 1,15 & 1,90 & 3,23 \\
\hline aids** & 110,3 & 0,65 & 0,51 & 0,33 \\
\hline câncer de esôfago & 2,7 & 1,93 & 2,29 & 2,14 \\
\hline câncer de estômago & 7,0 & 1,28 & 1,23 & 1,30 \\
\hline câncer de cólon & 4,1 & 0,79 & 0,95 & 0,40 \\
\hline câncer de fígado & 3,5 & 1,02 & 0,73 & 0,64 \\
\hline câncer de laringe & 2,5 & 1,68 & 1,99 & 1,51 \\
\hline câncer de pulmão & 15,6 & 0,78 & 0,79 & 0,70 \\
\hline diabetes & 8,3 & 1,31 & 1,37 & 1,27 \\
\hline doença reumática do coração & 1,7 & 0,94 & 1,18 & 1,31 \\
\hline doenças hipertensivas** & 5,1 & 1,77 & 2,08 & 3,02 \\
\hline infarto agudo miocárdio & 55,4 & 1,05 & 1,06 & 1,10 \\
\hline outras doenças isquêmicas do coração & o 13,0 & 0,85 & 0,84 & 0,87 \\
\hline doenças circ. pulm. e outras d. cor.** & 19,9 & 1,22 & 1,39 & 1,58 \\
\hline miocardiopatias** & 4,1 & 1,74 & 2,14 & 2,50 \\
\hline insuficiência cardíaca** & 4,9 & 1,62 & 1,76 & 2,15 \\
\hline doenças cerebrovasculares** & 22,5 & 1,51 & 1,86 & 2,34 \\
\hline pneumonias $* *$ & 20,7 & 1,39 & 1,45 & 1,50 \\
\hline doenças pulmonares obstrutivas & 7,3 & 1,19 & 1,23 & 1,37 \\
\hline alcoolismo** & 6,2 & 1,41 & 2,07 & 2,41 \\
\hline doença crônica do fígado e cirrose ${ }^{* *}$ & 19,6 & 1,43 & 1,41 & 1,48 \\
\hline insuficiência renal & 5,1 & 0,95 & 0,93 & 1,09 \\
\hline acidentes de trânsito & 38,7 & 1,02 & 1,10 & 1,19 \\
\hline afogamentos $^{* *}$ & 3,0 & 1,82 & 2,83 & 3,78 \\
\hline suicídios & 13,8 & 0,76 & 0,64 & 0,71 \\
\hline homicídios** & 55,4 & 1,58 & 1,71 & 2,95 \\
\hline
\end{tabular}

Fonte dos dados brutos: Fundação SEADE e IBGE

Raw data source: Fundação SEADE and IBGE

* coeficientes de mortalidade de 15 a 64 anos, por 100.000 habitantes padronizados por idade pelo método direto, usando como padrão a população total do município em 1991

*15 to 65 age group mortality rate per 100,000 inhabitants, using the direct method of age standardization, and the total 1991 population of the city as standard

** coeficientes de mortalidade com diferença estatisticamente significantes entre as áreas 4 e 1.

**mortality rates with statistically significant differences between areas 4 and 1

pecialmente pela concentração das emissões dos veículos automotores. Esses resultados sugerem a existência de algumas determinações relacionadas às condições gerais de vida que atuam em prejuízo de subpopulações que, em média, possuem as melhores condições socioeconômicas.

Por outro lado, a análise da maior parte das causas de morte revela expressiva dife- renciação com prejuízo dos grupos sociais residentes nas áreas de piores condições socioambientais, confirmando padrões observados em outros estudos ${ }^{12,13,14}$ e permitindo aprofundar e especificar os achados para o município de São Paulo.

Várias "explicações" para as diferenças sociais observadas na saúde têm sido avançadas; as diferenças poderiam ser o resul- 
Tabela 7 - Coeficientes de mortalidade* da área 1 e riscos relativos entre coeficientes* das áreas homogêneas 2, 3 e 4 em relação aos da área 1, segundo causas básicas, no sexo feminino.

Município de São Paulo, 1990-92.

Table 7 - Ratios of the mortality rates for homogeneous areas 2, 3 and 4 to the rate for area 1, by causes of death (female 15-64). São Paulo City, 1990-92.

\begin{tabular}{|c|c|c|c|c|}
\hline & área 1 & área 2 & área 3 & área 4 \\
\hline causa de morte & coeficiente & $\mathrm{rr}$ & $\mathrm{rr}$ & $\mathrm{rr}$ \\
\hline todas as causas & 209,9 & 1,11 & 1,24 & 1,53 \\
\hline tuberculose ${ }^{* *}$ & 1,1 & 1,92 & 2,78 & 3,51 \\
\hline doença de Chagas** & 1,2 & 1,50 & 2,64 & 4,27 \\
\hline aids & 9,8 & 0,95 & 0,89 & 0,75 \\
\hline câncer de esôfago & 0,5 & 0,78 & 1,83 & 2,13 \\
\hline câncer de estômago & 2,2 & 1,60 & 2,07 & 2,18 \\
\hline câncer de cólon & 3,1 & 1,12 & 0,69 & 0,62 \\
\hline câncer de fígado & 0,7 & 2,20 & 2,20 & 2,74 \\
\hline câncer de laringe & & $0,2 * * *$ & 2,63 & 3,81 \\
\hline câncer de pulmão & 4,5 & 0,80 & 0,87 & 0,88 \\
\hline câncer de mama & 18,2 & 1,05 & 0,77 & 0,75 \\
\hline câncer de útero porção não esp & 1,8 & 1,11 & 1,76 & 1,66 \\
\hline câncer de colo uterino & 3,4 & 1,22 & 1,20 & 1,92 \\
\hline diabetes** & 4,4 & 1,54 & 2,32 & 2,83 \\
\hline doença reumatica do coração & 1,9 & 0,98 & 1,44 & 1,81 \\
\hline doenças hipertensivas ${ }^{* *}$ & 4,1 & 1,42 & 1,70 & 2,87 \\
\hline infarto agudo miocárdio** & 13,4 & 1,35 & 1,56 & 2,02 \\
\hline outras doenças isquêmicas coração & 3,5 & 0,97 & 1,42 & 1,62 \\
\hline doenças circ. pulm. e out. d. cor.** & 10,1 & 1,31 & 1,69 & 2,21 \\
\hline miocardiopatias & 4,6 & 0,76 & 1,06 & 1,22 \\
\hline insuficiência cardíaca** & 2,9 & 1,52 & 2,06 & 2,74 \\
\hline doenças cerebrovasculares ${ }^{* *}$ & 18,0 & 1,07 & 1,55 & 2,26 \\
\hline pneumonias** & 7,8 & 1,08 & 1,36 & 1,80 \\
\hline doenças pulmonares obstrutivas & 4,0 & 1,02 & 1,42 & 1,78 \\
\hline alcoolismo & 0,5 & 1,94 & 2,54 & 3,15 \\
\hline doença crônica do fígado e cirrose & 3,6 & 1,16 & 1,50 & 1,82 \\
\hline insuficiência renal & 2,7 & 1,09 & 1,06 & 1,54 \\
\hline acidentes de trânsito & 9,2 & 0,96 & 1,05 & 1,13 \\
\hline afogamentos & 0,4 & 1,36 & 1,81 & 2,00 \\
\hline suicídios & 4,6 & 0,69 & 0,57 & 0,54 \\
\hline homicídios & 5,3 & 1,09 & 1,13 & 1,69 \\
\hline
\end{tabular}

Fonte dos dados brutos: Fundação SEADE e IBGE

Raw data source: Fundação SEADE and IBGE

* coeficientes de mortalidade de 15 a 64 anos, por 100.000 habitantes padronizados por idade pelo método direto, usando como padrão a população total do município em 1991

* 15 to 65 age group mortality rate per 100,000 inhabitants, using the direct method of age standardization, and the total 1991 population of the city as standard

** coeficientes de mortalidade com diferença estatisticamente significantes entre as áreas 4 e 1.

**mortality rates with statistically significant differences between areas 4 and 1

*** Como não ocorreram mortes em residentes da área 1 por câncer de laringe, o risco padrão considerado foi o da área 2 , cuja casela está preenchida com o coeficiente.

${ }_{* * *}$ As there were no deaths in area 1 residents due to larynx cancer, area 2 was considered for standard risk purposes. Area 2 presents the coefficient. 
tado de: artefatos (problemas no numerador/denominador, fonte de dados, tamanho de estratos etc.), viés de seleção (mobilidade social descendente em decorrência de doença), diferentes padrões de comportamento ou propriamente diferenças socioeconômicas e qualidade de vida ${ }^{6}$. Link e Phelan ${ }^{28}$ propõem o conceito de "causas sociais fundamentais" para entender as desigualdades sociais em saúde e o processo em que "as sociedades conformam padrões de doença através dos tempos". "As we define it, a fundamental social cause involves resources like knowledge, money, power, prestige, and social connections that strongly influence people's ability to avoid risks and to minimize the consequences of disease once it occurs. Because of the very general utility of these social and economic resources, fundamental causes are linked to multiple disease outcomes through multiple risk-factor mechanisms."

A explicação das desigualdades observadas no perfil da mortalidade do adulto em São Paulo deve considerar diferentes dimensões de determinantes. Uma primeira dimensão refere-se à prevalência e distribuição de fatores de risco. A intensa produção epidemiológica tem resultado na identificação de inúmeros fatores de risco, especialmente para as doenças crônicas não-transmissíveis. As doenças do aparelho circulatório e os cânceres, entre outras, têm tido sua ocorrência atribuída em maior ou menor grau ao aumento da exposição a agentes ambientais nocivos e a comportamentos não-saudáveis relacionados aos estilos de vida. O consumo de fumo, álcool, nutrição inadequada e sedentarismo têm sido considerados os mais importantes fatores de risco para esse conjunto de doenças. Diversos estudos mostram que a prevalência desses fatores se diferencia segundo os grupos sociais, marcadamente em prejuízo das populações com menores níveis de escolaridade e renda ${ }^{29,30}$. Portanto, as diferenças sociais observadas na mortalidade poderiam decorrer, em parte, das diferentes prevalências de fatores de risco. Entretanto, a distribuição diferenciada dos fatores de risco e estilos de vida não resulta de livres opções individuais, uma vez que se relacionam aos diferentes padrões de consumo social e cultural dos vários segmentos da população. Além disso, como foi discutido por Rose ${ }^{31}$, a estratégia preventiva com base na identificação e seguimento de indivíduos de alto risco é insuficiente, na medida em que não busca modificar o perfil de risco de toda a população.

Um conjunto de determinantes ainda pouco estudado no Brasil diz respeito aos efeitos, diferenciados socialmente, da assistência à saúde na chance de sobrevivência das pessoas acometidas ${ }^{15,32,33}$. Algumas causas de morte podem ser mais diretamente relacionadas com a baixa qualidade da assistência à saúde ou à falta de acesso a ela. Podem-se destacar entre essas causas as mortes por doenças hipertensivas e cerebrovasculares, doenças reumáticas do coração, diabetes, cânceres de colo do útero, doença de Chagas e aids. O diferente acesso a exames complementares, às terapêuticas mais adequadas, a serviço de saúde de qualidade na rotina e nas emergências ou à tecnologia de alto custo pode estar determinando parte das desigualdades reveladas neste estudo.

A abordagem dos determinantes das mortes de adultos em São Paulo precisa considerar, ainda, a análise das condições relacionadas ao ambiente, entendido num sentido amplo. Para a saúde do adulto importam, além dos aspectos de saneamento urbano e outros relativos à facilidade de disseminação de doenças infecciosas, os riscos ocupacionais e os decorrentes das condições de vida urbana "moderna" 34,35 . Doenças como a tuberculose, as pulmonares obstrutivas, algumas neoplasias e doenças cardiovasculares, bem como os acidentes de trabalho e os acidentes de trânsito, podem ter sua incidência facilitada por riscos existentes no ambiente de trabalho e vida urbanos.

A discussão dos determinantes relacionados ao ambiente remete ao impacto coletivo decorrente dos modelos de desenvolvimento e sua gestão, vigentes no município de São Paulo, e o acesso diferenciado 
dos diversos grupos sociais, às intervenções voltadas a melhoria da qualidade ambiental. A gestão do trânsito, da poluição, da habitação, dos espaços de lazer ou do trabalho necessitam da formulação de políticas setoriais e globais visando a promoção da qualidade de vida e a redução das desigualdades. A gestão da cidade pode ser planejada na direção de uma vida saudável para todos. Diversas experiências urbanas têm mostrado que o planejamento e a gestão ambiental das cidades podem priorizar a qualidade de vida da população $0^{36}$. Os riscos geradores dos altos níveis e dos diferenciais detectados na mortalidade não são inerentes ao desenvolvimento econômicosocial $^{37}$. Modelos aéticos, competitivos e segregadores trazem riscos adicionais, desnecessários e inaceitáveis para a vida das populações. A promoção da saúde, incorporando estratégias de modificação do perfil dos riscos, pode ser priorizada na formulação e execução das políticas sociais.

A análise das diferenças sociais da mortalidade na metrópole de São Paulo deve considerar as conseqüências estruturais das opções de desenvolvimento que priorizam o econômico em detrimento do social e favorecem a contínua concentração de renda e o impacto da miséria e da exclusão social na saúde das populações adultas do município. A violência, a desagregação e a exclusão social são componentes já estruturais do município. As políticas econômi- cas adotadas levam à situação de "irrelevância social" uma grande parcela da população, "desnecessária" ao modelo de desenvolvimento vigente ${ }^{38}$. $\mathrm{O}$ aumento das populações de rua, das favelas e dos trabalhadores informais são algumas das conseqüências do desemprego, da intensa concentração de renda e da redução salarial, expressando a ação do modelo econômico existente no país, que se reflete na vida da cidade. A desesperança define e conduz para opções de vida na exclusão, em que a cidadania inexiste. O homicídio é uma marca desse percurso de negação.

A identificação dos grupos de agravos mais freqüentes e dos seus possíveis determinantes ajuda a apontar, por um lado, a necessidade de medidas específicas de intervenção e, por outro, a necessidade de uma agenda inter-setorial, entre instâncias de governo, com planejamentos de prazos diferenciados, de estabelecimento de metas e avaliação permanente para um enfrentamento das iniqüidades verificadas. Como um dos esforços na busca da qualidade de vida, a epidemiologia pode aprofundar o conhecimento sobre os padrões e determinantes das condições de vida e saúde das populações, contribuindo para que a sociedade possa abrir canais democráticos de participação e discussão da gestão das cidades e do modelo econômico nacional, visando a redução das iniqüidades sociais.

\section{Summary}

Mortality in adulthood has been insufficiently studied in developing countries. The reduction in infant death rates and in mortality due to infectious diseases, among other factors, has led to an increased importance of adult mortality. In developing countries social inequalities in death rates of adults have been assessed less than in other age groups. The aim of this article is to analyze the social differences in mortality of adulthood in the City of São Paulo, from 1990-92. The urban area of the city was divided into four areas, which were considered as socioeconomically homogeneous. The division was based on socioeconomic variables obtained from the demographic census. People living in area 4 (the most underprivileged area) have higher mortality rates than those living in area 1 (the better off area). The social differences in mortality were higher in females than in males. In women, the mortality rate ratios between 
areas 4 and 1 were higher after the age of 25 , whilst in men the higher ratio was observed in the youngest age group (15-24 years). In area 1 the main basic cause of death in men was aids (17.4\% of all deaths). In area 4 the main cause was homicide (23.8\% of all deaths). In women the main cause in area 1 was breast cancer (9.1\%) whilst in area 4 it was cerebrovascular disease (12.3\%). In women, death rates for the following causes were 2 to 3 times higher in area 4 compared to area 1: Chagas disease, hypertension, cerebrovascular disease, pneumonia, coronary heart disease, diabetes and tuberculosis. In men: Chagas disease, hypertension, cerebrovascular disease, pneumonia, homicide, drowning, liver disease, cirrhosis and alcoholism. In males, as an exception, death rates from aids were lower in area 4 compared to area 1. The reasons for the observed differences should consider: a different prevalence of known risk factors for chronic diseases; different access to adequate medical care; different quality of urban environment and different socioeconomic levels of the population living in different areas of the city. In conclusion, this article showed different patterns of mortality in people living in different areas of City of São Paulo, and that the ecological approach seems to be an appropriate strategy to assess social differences in mortality among adults.

\section{Referências}

1. Hansluwka H. Some aspects of adult mortality in developed countries. Cah Soc Démogr Méd 1992; 31: 5-50.

2. Wigle DT, Mao R, Semenciw R, MC Cann C, Davies JW. Premature deaths in Canada: impact, trends and opportunities for prevention. Can J Public Health 1990; 81: 376-81.

3. Murray CJL, Feachem RGA. Adult mortality in developing countries. Trans R Soc Trop Med Hyg 1990; 84: 1-2.

4. Paim JS, Costa MCN, Cabral V, Mota IA, Neves RBB. Spacial distribution of proportional infant mortality and certain socioeconomics variables in Salvador, Bahia, Brazil. Bull Pan Am Health Organ 1987; 21: 225-39.

5. Breilh J, Granda E, Campaña A, Betancourt O. Ciudad y muerte infantil. Investigación sobre el deterioro de la salud en el capitalismo atrasado: un método. Quito: CEAS; 1987.

6. Black D, Morris JN, Smith C, Townsend P. Inequalities in health - The Black Report. London: Penguin Books; 1980.

7. Diderichsen F. Health and social inequities in Sweden. Soc Sci Med 1990; 31: 359-67.

8. Lehmann P, Mamboury C, Minder CE. Health and social inequities in Switzerland. Soc Sci Med 1990; 31: 369-86.

9. Valkonen T. Problems in the measurement and international comparisons of socio-economic differences in mortality. Soc Sci Med 1993; 36: 409-18.
10. Westerling R, Gullberg A, Rosén M. Socioeconomic differences in "avoidable" mortality in Sweden 19861990. Int J Epidemiol 1996; 25: 560-7.

11. Whitehead M. The health divide. London: Penguin Books; 1992.

12. Townsend $P$, Phillimore $P$, Beattie A. Health and deprivation: inequality and the north, Croom Helm, 1988 apud Whitehead M. The health divide. London: Penguin Books; 1992

13. Keil JE, Sutherland SE, Knapp RG, Tyroler HA. Does equal socioeconomic status in black and white men mean equal risk of mortality? Am J Public Health 1992; 82: $1133-6$.

14. Stephens C, Timaeus I, Ackerman M, Avle S, Maia PB, Campanário P et al. Environment and health in developing countries: an analysis of intra-urban differentials using existing data. São Paulo: London School of Hygiene \& Tropical Medicine/Fundação SEADE; 1994.

15. Lessa I. Anos produtivos de vida perdidos no Brasil, por mortalidade cardiovascular. Bol Oficina Sanit Panam 1991; 110: 118-25.

16. Mello Jorge MHP. Mortalidade por causas violentas no município de São Paulo [Tese de Doutoramento]. São Paulo: Faculdade de Saúde Pública da USP; 1979.

17. Minayo MCS, Souza ER. Violência para todos. Cad Saúde Pública 1993; 9: 65-78. 
18. Barata RCB, Ribeiro MCSA, Guedes MBLS, Moraes JC. Intra-urban differentials in death rates from homicide in the city of São Paulo, Brasil, 1988-1994. Soc Sci Med 1998; 47: 19-23.

19. Barreto ML. Esquistossomose mansônica: distribuição da doença e organização social do espaço [Dissertação de Mestrado] Salvador: Faculdade de Medicina da UFBa; 1982.

20. Breilh J, Granda E, Campaña A, Yépez J, Páez R, Costales P. Deterioro de la vida: un instrumento para análisis de prioridades regionales en lo social y la salud. Quito: Corporación Editora Nacional / CEAS; 1990.

21. Paim JS. Abordagens teórico-conceituais em estudos de condições de vida e saúde: notas para reflexão e ação. In: Barata RB organizador. Condições de vida e situação de saúde. Rio de Janeiro: ABRASCO; 1997. p. 7-30.

22. Illsley R. Comparative review of sources, methodology and knowledge. Soc Sci Med 1990; 31: 229-36.

23. Armitage P. Statistical methods in medical research. Oxford: Blackwell Scientific Publications; 1971.

24. Rutstein DD, Berenberger W, Chalmers TC, Fishmen AP, Perrin EB. Measuring the quality of medical care. N Engl J Med 1976; 294: 582-8.

25. Luna EJA, Lira MMTA, Drumond Jr M, Moraes L, D'Angelo LA. AIDS mortality in the city of São Paulo Brazil. In: Abstract book of the $9^{\text {th }}$ International Conference of AIDS; 1993; Berlin. v. 2, abstr. 667.

26. Drumond Jr M. Vigilância da morte evitável: acesso rápido e descentralização das informações. In: Barreto ML, Almeida Filho N, Veras RP, Barata RB, organizadores. Epidemiologia, serviços e tecnologias em saúde. Rio de Janeiro: FIOCRUZ/ABRASCO; 1998. p. 93-105.

27. Barros MBA. As mortes por suicídio no Brasil. In: Cassorla RMS, coordenador. Do suicídio - estudos brasileiros. São Paulo: Papirus; 1991.

28. Link BG, Phelan JC. Understanding sociodemographic differences in health - the role of fundamental social causes [editorial]. Am J Public Health 1996; 86: 471-2.
29. Martins IS, Coelho LT, Matos IMS, Mazzilli RN, Trigo M, Wilson D. Dislipidemias e alguns fatores de risco associados em uma população periférica da Região Metropolitana de São Paulo, SP-Brasil: um estudo piloto. Rev Saúde Pública 1989; 23: 236-43.

30. Duncan BB, Schmidt M, Achutti A. et al. Prevalence and detection status of hypertention in a large Braziliam city: influence of social factors. [Presented at the $2^{\text {nd }}$ International Conference on Preventive Cardiology; 1989; Washington (DC)].

31. Rose G. Individuos enfermos e poblaciones enfermas. In: Organización Panamericana de la Salud. El desafio de la epidemiologia: problemas e lecturas selecionadas. Washington (DC); 1989. (OPS - Publicación Científica, 505).

32. Chor D, Fonseca MJM, Andrade CR, Waissmann W, Lotufo PA. Doenças cardiovasculares: panorama da mortalidade no Brasil. In: Minayo MCS, organizador. Os muitos Brasis. Saúde e população na década de 80. São Paulo: Hucitec/Rio de Janeiro: ABRASCO; 1995. p. 57-86.

33. Acúrcio FA, Cesar CC, Guimarães, MDC. Utilização de cuidados de saúde e sobrevida entre pacientes com AIDS em Belo Horizonte, Minas Gerais, Brasil. Cad Saúde Pública 1998; 14: 811-20.

34. Reichenheim ME, Werneck GL. Adoecer e morrer no Brasil dos anos 80: perspectivas de novas abordagens. In: Guimarães R, Tavares R, organizadores. Saúde e sociedade no Brasil: anos 80. Rio de Janeiro: Relume Dumará; 1994. p. 113-30.

35. Rogers RG, Hackenberg R. Extending epidemiologic transition theory: a new stage. Soc Biol 1987; 34: 234-43.

36. Mendes EV. Uma agenda para a saúde. São Paulo: Hucitec; 1996.

37. Kjellstron T, Rosenstock L. The role of environmental and occupational hazards in the adult health transition. World Health Stat Q 1990; 43: 188-96.

38. Ribeiro LCQ, Santos Jr OA. Desigualdade e exclusão. Rev Teoria Debate 1993; 20: 61-5. 